\title{
An evolutionary perspective on habenular asymmetry in humans
}

\author{
Miguel L. Concha', ${ }^{1,3 *}$, Patricio Ahumada-Galleguillos ${ }^{1,2}$ \\ 'Anatomy and Developmental Biology, Institute of Biomedical Sciences, Faculty of Medicine, Universidad de Chile, PO Box 70031, Santiago, Chile \\ ${ }^{2}$ Biomedical Neuroscience Institute, Independencia 1027, Santiago, Chile \\ ${ }^{3}$ Center for Geroscience, Brain Health and Metabolism, Santiago, Chile
}

\section{Article Info}

\section{Article Notes}

Received: October 22, 2016

Accepted: November 22, 2016

\section{*Correspondence:}

Dr. Miguel L. Concha,

Anatomy and Developmental Biology, Faculty of Medicine, Universidad de Chile, PO Box 70031, Santiago, Chile:

Email: mconcha@med.uchile.cl

C 2016 Concha ML. This article is distributed under the terms of the Creative Commons Attribution 4.0 International License

\section{Keywords:}

Habenula

Asymmetry

Structure

Function

Vertebrates

Humans

\section{ABSTRACT}

The habenula $(\mathrm{Hb})$ of vertebrates is a dorsal and bilateral diencephalic nuclear complex that works as an anatomical hub integrating cognitive, emotional and sensory networks to regulate mood, motivation and valuebased decision-making, among other functions. Across vertebrates, the $\mathrm{Hb}$ organises into two conserved separate components (medial and lateral in mammals equivalent to dorsal and ventral in more basal vertebrate species), which are thought to subserve different functions based on a partial independence of their connectivity systems. As a complex, the $\mathrm{Hb}$ shows morphological, molecular and connectivity differences between the left and right sides in a wide range of vertebrate species, which in some cases extend to the functional and behavioural levels. Habenular asymmetries are particularly prominent in basal vertebrate species but become less evident in amniotes and particular mammals. In humans, recent evidence reveals that, under an overall symmetry morphology, the Hb shows lateral differences in volume, activation, metabolism and susceptibility to damage that suggest an asymmetric condition of this nuclear complex. Here, we review the evidence supporting this view and discuss the possible origin of this asymmetric trait in humans from an evolutionary developmental perspective.

The habenula as a key regulator of mood, motivation and decision-making

In the brain of vertebrates, the habenula $(\mathrm{Hb})$ is a dorsal and bilateral diencephalic nuclear complex that works as an anatomical hub that integrates cognitive, emotional and sensory networks to regulate mood, motivation and value-based decision-making, among other processes. In mammals, the left and right sides of the $\mathrm{Hb}$ are formed by medial (Med-Hb) and lateral (Lat-Hb) components which are thought to subserve different functions based on a partial independence of their afferent and efferent connectivity systems (Figure 1A) ${ }^{1,2}$. The Med-Hb primarily receives inputs from the supracommissural septum and projects to the interpeduncular nucleus (IPN) in the ventral midbrain (Figure 1A, green) ${ }^{3}$. The Lat$\mathrm{Hb}$, on the other hand, has more widespread connectivity linking the basal ganglia and limbic forebrain with brainstem dopaminergic (substantia nigra pars compacta, SN; ventral tegmental area, VTA), serotonergic (raphe nuclei), histaminergic (hypothalamus) and gabaergic (rostro medial mesopontine tegmental nucleus, RMTg) centres (Figure 1A, red) ${ }^{4,5}$. Despite the significant amount of experimental data supporting the view of partial independence between the Med-Hb and Lat- $\mathrm{Hb}$, there is nevertheless some degree of overlapping of afferent projections (i.e. descendent afferents from the accumbens and diagonal band nuclei, and ascendant afferents 
(A)

$$
\text { CONNECTIVITY /FUNCTION }
$$

(B)

\section{ASYMMETRY}

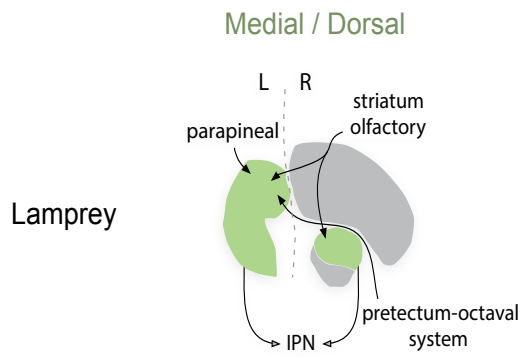

Lateral / Ventral
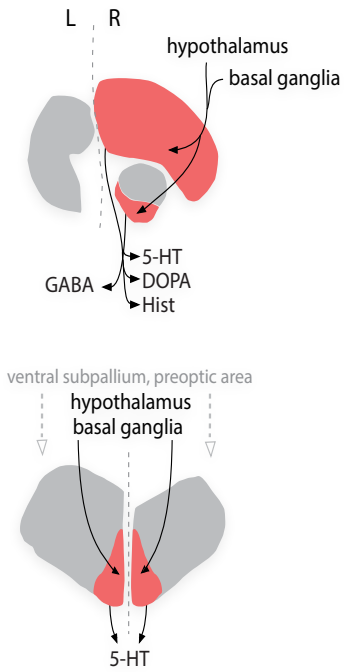

Conflict resolution of social aggression

Control of fear responses
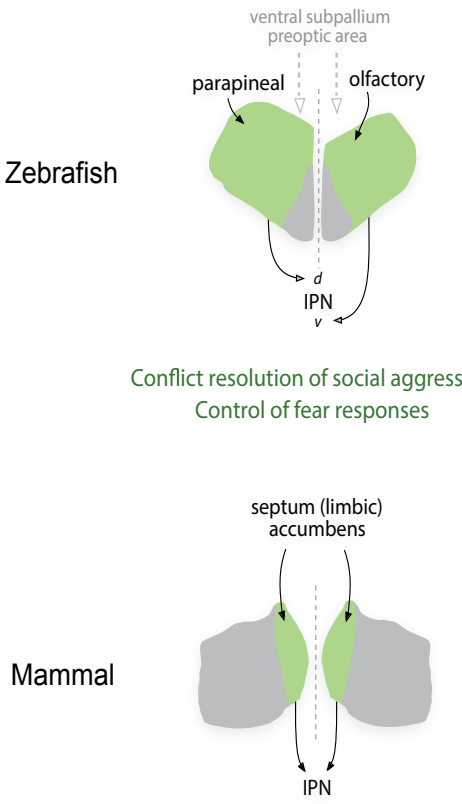

Depressive behaviours Fear memory expression and affective states

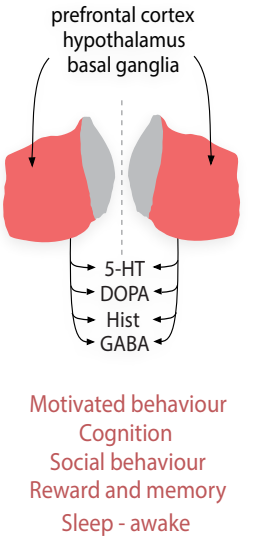

Size $R>L$

Domains Dorsal $L>R$; ventral only on $L$

Gene expression PitxA L; P-ERK R $>$ L; $k c t d 12 L>R$

Connectivity Parapineal and octaval to $L H b$
Size $L>R$

Domains Dorso-lateral $L>R$; dorso-medial $R>L$ Ventral symmetric?

Gene expression Nodal L; Brn3a R $>$ L; $k \operatorname{ctd} 12.1 \mathrm{~L}>\mathrm{R}$

Connectivity Parapineal to $\mathrm{L} \mathrm{Hb}$; olfactory to $\mathrm{R} \mathrm{Hb}$ $\mathrm{LR} \mathrm{Hb}$ efferents project to DV IPN

Neuronal activation $L>R$ to light, $R>L$ to olfactory stimulus

\author{
Size $L>R$ (humans) \\ Domains Lat- $H b L>R$ (humans), $R>L$ (mice) \\ Med-Hb L > R (rats) \\ Cell types Unique cell group on $L$ (macrosmatic mole) \\ Integrity Lateralised damage in depression (humans) \\ Functional connectivity Lateralised connectivity to VTA, SNc and \\ parahippocampus (humans) \\ Activation Anti-symmetric in postnatal stressed mice \\ Lateral activation in aversive conditions in \\ normal and depressive individuals (humans) \\ Metabolism Decreased $\mathrm{R}$ metabolism after ketamine in \\ depressive individuals (humans)
}

Figure 1: A comparative view of habenular connectivity, function and asymmetry in vertebrates.

(A) Organisation of connectivity and proposed function of the two main habenular pathways in vertebrates. Gene expression, neurochemistry and connectivity data from a basal vertebrate (lamprey), a modern teleost (zebrafish) and mammals (rodents and humans) support the existence of two partially independent habenular circuits. The medial habenular pathway of mammals is homologous to the dorsal habenular pathway of lampreys and zebrafish (left, green). On the other hand, the lateral habenular pathway of mammals is equivalent to the ventral habenular pathway of lampreys and zebrafish (right, red). The proposed motor-limbic and cognitive functions associated with each habenular domain are given below the schematics. (B) Left-right asymmetries of the $\mathrm{Hb}$ described at structural and functional levels in the lamprey, zebrafish and mammals (rodents and humans). Abbreviations: 5-HT (serotonergic), $d$ (dorsal), DOPA (dopaminergic), GABA (gabaergic), Hist (histaminergic), IPN (interpeduncular nucleus), L (left), R (right), v (ventral). Details and references are given in the text.

from the VTA and locus coeruleus) $)^{2,6}$ and interconnectivity ${ }^{7}$ between the two Hb domains.

Given the efferent connectivity pattern and the connection of its afferent septal nuclei with specific sectors of the hippocampus and subiculum, the mammalian Med$\mathrm{Hb}$ has been involved in learning associated with fear responses and mood and to nicotine addiction, among other functions ${ }^{3}$. In turn, studies in rats and monkeys have shown that projections from the Lat-Hb to the VTA and SN provide reward prediction error and value related signals to dopaminergic systems that are fundamental to learning ${ }^{8,9}$. Studies in humans support this view and reveal $\mathrm{Hb}$ activation and increased functional connectivity between the $\mathrm{Hb}$, VTA and SN in the context of reward 
predictor errors and negative motivation values associated with punishment ${ }^{10-13}$. In addition to learning, specific projections from the Lat-Hb to the serotonergic raphe nuclei provide long-term coding signals that appear to influence mood ${ }^{6,14}$. Consistently, structural and functional disruption of the Lat-Hb in humans has been linked to the origin of depression ${ }^{15-22}$ and substance abuse (cocaine and alcohol ${ }^{23,24}$. Furthermore, deep brain stimulation of the Lat-Hb has proven successful to alleviate depressive symptoms in patients with treatment-resistant major depression $^{25,26}$. Together, these findings reveal that as a complex, the $\mathrm{Hb}$ is an anatomical hub integrating cognitive, emotional and sensory networks and that in this context participates in the modulation of a series of motivated behaviours, including mood, learning and value-based decision-making.

\section{Evolution of habenular circuits in vertebrates}

Across vertebrates, the structural organisation of the $\mathrm{Hb}$ into two separate components seems to be an ancestral character, which is also closely related to the conservation of two segregated systems of habenular connectivity ${ }^{27-29}$. Particularly, the primary outputs to the IPN and to dopaminergic/serotonergic systems that characterise the medial and lateral domains of the mammalian $\mathrm{Hb}$, respectively, ${ }^{2}$ are also present in the dorsal and ventral habenular domains of more basal vertebrate species such as lampreys and zebrafish (Figure $1 \mathrm{~A})^{28-31}$. However, such conservation of the output circuit design differs from the evolutionary shift of inputs from sensorial (e.g. olfactory, parapineal, octaval system) to limbic, which is seen in the medial/dorsal habenular domain from basal vertebrates to mammals ${ }^{5,29,32-34}$. This observation suggests a change in the contextual information that is used by different species to activate habenular circuits. Also, in the evolution towards amniotes and specifically in mammals we observe a striking diversification of input sources to the Lat- $\mathrm{Hb}^{1,2}$, including the appearance of reciprocal projections from neuromodulatory monoaminergic habenular targets ${ }^{1,35}$. Interestingly, the relative size of the Lat-Hb is also significantly increased in humans compared with rats, such that it represents $\sim 95 \%$ of the total Hb volume ${ }^{36,37}$. Thus, there appears to be a growing diversification of afferent sources to the lateral/ventral habenular domain during evolution, in particular in mammals, which suggests an increasingly complex modulation of this habenular circuit. Although detailed studies of habenular connectivity in other vertebrate species are still needed, this idea finds support in the extensive range of functional/behavioural domains (i.e. sensorial, motivational and cognitive) in which the Lat-Hb has been involved in mammals and especially in humans ${ }^{5,9}$.
Asymmetry: a conserved but variable trait in the habenula of vertebrates

A noticeable and conserved feature of the bilateral $\mathrm{Hb}$ is the presence of morphological and molecular differences between the left and right sides (Figure 1B) ${ }^{27}$. Although studies on habenular asymmetry have comprised a wide range of vertebrate species, they have mostly referred to the $\mathrm{Hb}$ as a complex and little information is available on the contribution of the medial/dorsal and lateral/ventral components to habenular asymmetry across species. As a complex, habenular asymmetry is morphologically conspicuous in agnathans and most cartilaginous and bony fishes ${ }^{27}$ and can involve both medial and lateral domains (e.g. lampreys) ${ }^{29}$ or be confined to a single domain (e.g. medial/dorsal $\mathrm{Hb}$ zebrafish) ${ }^{30}$. In vertebrate species other than fishes, and in particular in amniotes such as birds and mammals, the morphological asymmetries of the $\mathrm{Hb}$ are much less pronounced with only a few exceptions (e.g. lizards) ${ }^{27}$. In mammals, the presence of small lateral differences in habenular volume has been described in the Med-Hb of rats $^{38}$ and the Lat-Hb of mice ${ }^{39}$, while a unique small group of cells was described on the left $\mathrm{Hb}$ of the macrosmatic mole ${ }^{40}$. In primates and in particular in humans, the study of habenular asymmetry is still in its infancy due to the small size and internal position of this nuclear complex within the brain. In spite of this, a recent post-mortem volumetric analysis revealed that the human $\mathrm{Hb}$ is significantly larger on the left compared to the right in both genders, a condition that results from an enlargement of the left Lat- $\mathrm{Hb}^{37}$. Consistent with these findings, a volumetric study using high-resolution magnetic resonance imaging (MRI) showed a tendency of increased left habenular volume in healthy subjects and individuals with various neuropsychiatric conditions, although these differences were statistically non-significant possibly due to the resolution limitations of this imaging technique ${ }^{17}$.

Asymmetry of the $\mathrm{Hb}$ does not restrict to the morphological and molecular domains but has also been observed at a functional level, suggesting that the $\mathrm{Hb}$ is involved in brain lateralisation. In zebrafish, for example, specific subsets of neurones on the dorsal $\mathrm{Hb}$ show differential calcium activation on the left and right sides in response to visual and olfactory stimuli, respectively ${ }^{41}$. Importantly, these functional asymmetries match the structural asymmetries in habenular morphology, gene expression and connectivity ${ }^{30,41}$, which have also been associated with visual-guided lateralised behaviours ${ }^{42}$. In mammals, recent studies reveal that the left and right $\mathrm{Hb}$ become differentially activated under specific conditions, but the anatomical substrate underlying habenular asymmetric activation is still unknown. In mice, the $\mathrm{Hb}$ becomes asymmetrically activated in postnatal mice under stress as revealed by the expression of early response genes 
in a transgenic background ${ }^{43}$. In humans, studies of highresolution functional MRI have shown asymmetric blood oxygen level-dependent responses to aversive relative to neutral events ${ }^{13}$ and to negative conditioned stimulus values $^{12}$. Furthermore, a recent report based on highresolution cardiac-gated resting state imaging revealed that the right and left $\mathrm{Hb}$ have distinct correlates of functional connectivity with the VTA, SN and the parahippocampus ${ }^{44}$. In turn, studies in depressive patients show a significant decrease in activation of the left $\mathrm{Hb}$ during the prediction or experience of monetary penalty ${ }^{45}$, a decreased metabolism of the right $\mathrm{Hb}$ after ketamine treatment ${ }^{18}$, and a reduction of volume, neuronal cell number and cell area in histological postmortem samples ${ }^{16}$. Together, these findings suggest that the human $\mathrm{Hb}$ has a previously unrecognised asymmetric condition that comprises both structural and functional levels, which might be relevant in contexts of health and disease. In summary, morphological, molecular and connectional observations across vertebrates reveal the presence of notorious asymmetries in more basal species, which in some cases is linked to the asymmetric response of the $\mathrm{Hb}$ to sensorial stimuli and lateralised behaviours. In contrast, in the context of subtle morphological asymmetries, the $\mathrm{Hb}$ of mammals and particularly humans often shows asymmetries at a functional level but the anatomical substrate underlying this condition remains unknown.

\section{Coding asymmetries on habenular circuits}

From a functional viewpoint, a relevant question is how the different types of asymmetries are coded into circuits. Asymmetric circuits involving paired neuronal structures such as the $\mathrm{Hb}$ can in principle use two main designs: exhibit left-right differences in the extent of similar types of neurones/connectivity present on left and right sides (class-I), or show unique neurones or connectivity patterns on only one side of the brain (class-II) ${ }^{46}$. The Hb appears to shows both types of circuit designs in vertebrates. For example, the left and right $\mathrm{Hb}$ of a modern teleost such as zebrafish exhibit different proportions of similar neurones based on molecular markers and connectivity ${ }^{30}$. The presence of volumetric differences between the left and right sides of the $\mathrm{Hb}$ in humans ${ }^{37}$ and many other vertebrate species ${ }^{27}$ could also be indicative of a class-I asymmetry. On the other hand, the $\mathrm{Hb}$ of lampreys, frogs and lizards show unilateral cellular domains, i.e. the ventral $\mathrm{Hb}$ of lampreys is only on the right side ${ }^{29}$ while the laterodorsal and dorsomedial $\mathrm{Hb}$ domains of frogs ${ }^{47}$ and lizards ${ }^{48}$, respectively, are present only on the left ${ }^{27}$. On the other hand, a group of neurones of the olfactory bulb project only to the right $\mathrm{Hb}$ in zebrafish ${ }^{33,34}$ while parapineal neurones reach only the left $\mathrm{Hb}$ in lampreys, zebrafish and lizards ${ }^{32,48,49}$. Noteworthy, the observation of sided activation of the human $\mathrm{Hb}$ in certain functional tasks, the unilateral metabolic response to drugs and lateral damage in certain neuropsychiatric conditions (see above) suggest the presence of class-II asymmetric circuits in the human $\mathrm{Hb}$.

In the context of the different asymmetric habenular designs, some elaborate patterns of connectivity have emerged. A striking example is provided by zebrafish, where the spatial segregation of left-right habenular outputs along the dorsoventral axis of the IPN define parallel tunnels of habenular information directed to the griseum centrale and raphe nuclei, respectively ${ }^{30,50}$. Significantly, habenular asymmetry and its coding into segregated efferent connectivity tunnels have been associated in this species with the asymmetric processing of visual and olfactory stimuli ${ }^{41,51}$, lateralised behaviours ${ }^{42}$, and the behavioural responses to fear and anxiety ${ }^{50,52}$. Whether similar asymmetric patterns of functional connectivity and behavioural correlates are associated with the $\mathrm{Hb}$ of other vertebrate species including humans is yet to be determined.

\section{Insights into the origin and impact of habenular asymmetry in humans}

An important lesson from the comparative studies described here is the existence of multiple types of habenular asymmetries in different species affecting various organisational levels, from gene expression through morphology and connectivity to function. In this context, it does not appear sensible to provide a single explanation for the origin of habenular asymmetry in humans. On a developmental ground, studies in fishes have suggested that the Hb shows independent developmental programs on the left and right sides that can be modulated unilaterally by genetic and cellular inputs to generate asymmetry during ontogeny ${ }^{53,54}$. For example, the leftsided activation of the transforming growth factor- $\beta$ Nodal in the embryonic brain controls for development of morphological, molecular and connectional asymmetries in lampreys, catshark and zebrafish ${ }^{53,55,56}$. Nodal signalling is a conserved pathway that regulates the development of body, visceral and brain asymmetries across metazoans in a context-dependent manner ${ }^{54,57,58}$, and in vertebrates, it has been associated with both enlarged and reduced habenular domains on the side of expression ${ }^{53,55,56}$. In addition to direct genetic inputs, the influence of an asymmetrically positioned cellular group, the parapineal organ ( $\mathrm{PpO}$ ), also plays a fundamental role in the elaboration of habenular asymmetries in zebrafish ${ }^{32,59,60}$. Notably, genetic (Nodal) and cellular ( $\mathrm{PpO}$ ) influences upon the developing $\mathrm{Hb}$ both seem to converge in the regulation of neurogenesis ${ }^{56,61}$. It is thus plausible that an asymmetric regulation of neurogenesis (via Nodal and other developmental inputs) is a conserved step in the origin of habenular asymmetry across vertebrates including humans. This idea is 
consistent with the reduction or evolutionary loss of gross morphological asymmetries observed in vertebrate groups other than fishes including mammals ${ }^{27}$, which follows the apparent loss of Nodal expression in the brain ${ }^{54}$. The mechanisms controlling habenular asymmetry in the absence of asymmetric Nodal signalling possibly exploit the sensitivity of the prospective $\mathrm{Hb}$ to spatial and temporal changes in genetic and cellular developmental inputs, as suggested by zebrafish studies ${ }^{32,60-63}$. These mechanisms could in principle generate structural and functional asymmetries in mammals beneath an apparent overall symmetric morphology of the $\mathrm{Hb}$. The existence of a unique small group of cells on the left $\mathrm{Hb}$ of the macrosmatic mole $^{40}$, of lateral differences in volume described in the $\mathrm{Hb}$ of rodents and humans ${ }^{37-39}$, of asymmetric functional connectivity in humans ${ }^{44}$, and asymmetric activation of the rodent and human $\mathrm{Hb}$ in different contexts of health and disease $\mathrm{e}^{12,13,43,45}$ is consistent with this idea and supports the importance of considering other less evident types of habenular asymmetries in these species.

Functional studies involving areas such as the prefrontal cortex, amygdala, cerebellum and mesencephalon (i.e. optic tectum or colliculi) have shown that lateralisation is a key feature of the human brain ${ }^{64}$. In this context, demonstrating the existence of asymmetric circuit designs involving the human $\mathrm{Hb}$ is relevant to first understand the possible functional role of habenular asymmetry and then link this condition to a potential therapeutic use in the treatment of affective disorders. In contrast to sensorial asymmetries that in many cases associate with lateralised environmental stimuli, the mammalian $\mathrm{Hb}$ primarily integrates cognitive with sensorial and motivational information to generate an activation state that is contextually dependent, and thus the role of asymmetry in this process is less intuitive. The partial independence of left and right habenular circuits provides a substrate that could promote and perhaps explain the presence of sided activation and lateral susceptibility of the Hb to damage seen in humans. Should this is the case, it will become essential to distinguish the functions that the left and right $\mathrm{Hb}$ do in common from those that are unique to each of them. Future research will have to address these and other emerging questions and ascertain the presence and types of left-right asymmetric circuits in the human $\mathrm{Hb}$ and their correlates to habenular and brain function. To this aim, special efforts must be placed to improve the resolution of in vivo functional imaging and segmentation techniques, and to develop novel experimental designs that resolve left from right in the human $\mathrm{Hb}$.

\section{Acknowledgements}

We thank Karina Palma for drawing the panels of the figure, and Iskra Signore for critical reading of the manuscript. This work was supported by the National Commission for Scientific and Technological Research
(FONDECYT 1020902 and 3160486 to M.L.C and P.A.G., respectively; Ring Initiative PIA ACT1402 to M.L.C; Fondap 15150012 to M.L.C.), and the Millennium Science Initiative (P09-015-F to M.L.C).

\section{References}

1. Sutherland RJ. The dorsal diencephalic conduction system a review of the anatomy and functions of the habenular complex. Neuroscience Biobehavioural Review. 1982; 6(1): 1-13.

2. Bianco IH, Wilson SW. The habenular nuclei: a conserved asymmetric relay station in the vertebrate brain. Philos Trans R Soc Lond B Biol Sci. 2009; 364(1519): 1005-1020.

3. Viswanath $\mathrm{H}$, Carter $\mathrm{AQ}$, Baldwin PR, et al. The medial habenula still neglected. Front Hum Neurosci. 2013; 7: 931.

4. Lecourtier L, Kelly PH. A conductor hidden in the orchestra Role of the habenular complex in monoamine transmission and cognition. Neurosci Biobehav Rev. 2007; 31(5): 658-672.

5. Hikosaka 0. The habenula: from stress evasion to value-based decision-making. Nat Rev Neurosci. 2010; 11(7): 503-513.

6. Boulos LJ, Darcq E, Kieffer BL. Translating the Habenula-From Rodents to Humans. Biol Psychiatry. 2016; http://dx.doi.org/10.1016/j. biopsych.2016.06.003.

7. Kim U, Chang SY. Dendritic morphology local circuitry and intrinsic electrophysiology of neurons in the rat medial and lateral habenular nuclei of the epithalamus. Journal of Comparative Neurology. 2005; 483(2): 236-250.

8. Matsumoto M, Hikosaka 0 . Lateral habenula as a source of negative reward signals in dopamine neurons. Nature. 2007; 447(7148): 11111115.

9. Namboodiri VM, Rodriguez-Romaguera J, Stuber GD. The habenula. Curr Biol. 2016; 26(19): R873-R877.

10. Salas R, Baldwin P, de Biasi M, et al. BOLD Responses to Negative Reward Prediction Errors in Human Habenula. Front Hum Neurosci. 2010; 4: 36.

11. Ide JS, Li CS. Error-related functional connectivity of the habenula in humans. Front Hum Neurosci. 2011; 5: 25

12. Lawson RP, Seymour B, Loh E, et al. The habenula encodes negative motivational value associated with primary punishment in humans. Proc Natl Acad Sci U S A. 2014; 111(32): 11858-11863.

13. Hennigan K, D'Ardenne K, McClure SM. Distinct midbrain and habenula pathways are involved in processing aversive events in humans. J Neurosci. 2015; 35(1): 198-208.

14. Proulx CD, Hikosaka 0 , Malinow R. Reward processing by the lateral habenula in normal and depressive behaviors. Nat Neurosci. 2014;17(9): 1146-1152.

15. Morris JS, Smith KA, Cowen PJ, et al. Covariation of activity in habenula and dorsal raphe nuclei following tryptophan depletion. Neuroimage. 1999; 10(2): 163-172.

16. Ranft K, Dobrowolny H, Krell D, et al. Evidence for structural abnormalities of the human habenular complex in affective disorders but not in schizophrenia. Psychol Med. 2010; 40(4): 557-567.

17. Savitz JB, Nugent AC, Bogers W, et al. Habenula volume in bipolar disorder and major depressive disorder a high-resolution magnetic resonance imaging study. Biol Psychiatry. 2011; 69(4): 336-343.

18. Carlson PJ, Diazgranados N, Nugent AC, et al. Neural correlates of rapid antidepressant response to ketamine in treatment-resistant unipolar depression a preliminary positron emission tomography study. Biol Psychiatry. 2013; 73(12): 1213-1221.

19. Carceller-Sindreu M, de Diego-Adelino J, Serra-Blasco M, et al. 
Volumetric MRI study of the habenula in first episode recurrent and chronic major depression. Eur Neuropsychopharmacol. 2015; 25(11): 2015-2021.

20. Johnston BA, Steele JD, Tolomeo S, et al. Structural MRI-Based Predictions in Patients with Treatment-Refractory Depression (TRD). PLoS One. 2015; 10(7): e0132958.

21. Ely BA, Xu J, Goodman WK, et al. Resting-state functional connectivity of the human habenula in healthy individuals: Associations with subclinical depression. Hum Brain Mapp. 2016; 37(7): 2369-2384.

22. Schmidt FM, Schindler S, Adamidis $M$, et al. Habenula volume increases with disease severity in unmedicated major depressive disorder as revealed by 7T MRI. Eur Arch Psychiatry Clin Neurosci. 2016; doi: 10.1007/s00406-016-0675-8.

23. Deserno L, Beck A, Huys QJ, et al. Chronic alcohol intake abolishes the relationship between dopamine synthesis capacity and learning signals in the ventral striatum. Eur J Neurosci. 2015; 41(4): 477-486.

24. Parvaz MA, Konova AB, Proudfit GH, et al. Impaired neural response to negative prediction errors in cocaine addiction. J Neurosci. 2015; 35(5): 1872-1879.

25. Sartorius A, Kiening KL, Kirsch P, et al. Remission of major depression under deep brain stimulation of the lateral habenula in a therapyrefractory patient. Biol Psychiatry. 2010; 67(2): e9-e11.

26. Kiening K, Sartorius A. A new translational target for deep brain stimulation to treat depression. EMBO Mol Med. 2013; 5(8):11511153.

27. Concha ML, Wilson SW. Asymmetry in the epithalamus of vertebrates. J Anat. 2001; 199(Pt 1-2): 63-84.

28. Amo R, Aizawa H, Takahoko $\mathrm{M}$, et al. Identification of the zebrafish ventral habenula as a homolog of the mammalian lateral habenula. J Neurosci. 2010; 30(4): 1566-1574.

29. Stephenson-Jones M, Floros O, Robertson B, et al. Evolutionary conservation of the habenular nuclei and their circuitry controlling the dopamine and 5-hydroxytryptophan (5-HT) systems. Proc Natl Acad Sci U S A. 2012;109(3): E164-173.

30. Aizawa H, Bianco IH, Hamaoka T, et al. Laterotopic representation of left-right information onto the dorso-ventral axis of a zebrafish midbrain target nucleus. Curr Biol. 2005;15(3): 238-243.

31. Turner KJ, Hawkins TA, Yanez J, et al. Afferent Connectivity of the Zebrafish Habenulae. Front Neural Circuits. 2016;10: 30.

32. Concha ML, Russell C, Regan JC, et al. Local tissue interactions across the dorsal midline of the forebrain establish CNS laterality. Neuron. 2003; 39(3): 423-438.

33. Hendricks M, Jesuthasan S. Asymmetric innervation of the habenula in zebrafish. J Comp Neurol. 2007; 502(4): 611-619.

34. Miyasaka N, Morimoto K, Tsubokawa T, et al. From the olfactory bulb to higher brain centers: genetic visualization of secondary olfactory pathways in zebrafish. J Neurosci. 2009; 29(15): 4756-4767.

35. Herkenham M, Nauta WJ. Efferent connections of the habenular nuclei in the rat. J Comp Neurol. 1979; 187(1): 19-47.

36. Diaz E, Bravo D, Rojas X,et al. Morphologic and immunohistochemical organization of the human habenular complex. J Comp Neurol. 2011; 519(18): 3727-3747.

37. Ahumada-Galleguillos P, Lemus CG, Diaz E, et al. Directional asymmetry in the volume of the human habenula. Brain Struct Funct 2016; doi: 10.1007/s00429-016-1231-z.

38. Wree A, Zilles K, Schleicher A. Growth of fresh volumes and spontaneous cell death in the nuclei habenulae of albino rats during ontogenesis. Anatatomy and Embryology. 1981; 161(4): 419-431.

39. Zilles K, Schleicher A, Wingert F. [Quantitative growth analysis of limbic nuclei areas fresh volume in diencephalon and mesencephalon of an albino mouse ontogenic series. III. Nucleus interpe-uncularis]. Journal fur Hirnforschung. 1976; 17(1): 21-29.

40. Kemali M. Morphological asymmetry of the habenulae of a macrosmatic mammal, the mole. Zeitschrift fur Mikroskopish Anatatomische Forschung. 1984; 98(6): 951-954.

41. Dreosti E, Vendrell Llopis N, Carl M, et al. Left-right asymmetry is required for the habenulae to respond to both visual and olfactory stimuli. Curr Biol. 2014; 24(4): 440-445.

42. Barth KA, Miklosi A, Watkins J,et al. fsi zebrafish show concordant reversal of laterality of viscera, neuroanatomy, and a subset of behavioral responses. Curr Biol. 2005; 15(9): 844-850.

43. Ichijo H, Hamada M, Takahashi S, et al. Lateralization, maturation, and anteroposterior topography in the lateral habenula revealed by ZIF268/EGR1 immunoreactivity and labeling history of neuronal activity. Neurosci Res. 2015; 95: 27-37.

44. Hetu S, Luo Y, Saez I, et al. Asymmetry in functional connectivity of the human habenula revealed by high-resolution cardiac-gated resting state imaging. Hum Brain Mapp. 2016; 37(7): 2602-2615.

45. Furman DJ, Gotlib IH. Habenula responses to potential and actual loss in major depression preliminary evidence for lateralized dysfunction. Soc Cogn Affect Neurosci. 2016; 11(5): 843-851.

46. Concha ML, Bianco IH, Wilson SW. Encoding asymmetry within neural circuits. Nat Rev Neurosci. 2012; 13(12): 832-843.

47. Braitenberg V, Kemali M. Exceptions to bilateral symmetry in the epithalamus of lower vertebrates. Journal of Comparative Neurology. 1970 ; 138(2): 137-146.

48. Engbretson GA, Reiner A, Brecha N. Habenular asymmetry and the central connections of the parietal eye of the lizard. Journal of Comparative Neurology. 1981;198(1):155-165.

49. Yañez J, Pombal MA, Anadon R. Afferent and efferent connections of the parapineal organ in lampreys: a tract tracing and immunocytochemical study. Journal of Comparative Neurology. 1999; 403(2): 171-189.

50. Agetsuma M, Aizawa H, Aoki T, et al. The habenula is crucial for experience-dependent modification of fear responses in zebrafish. Nat Neurosci. 2010; 13(11): 1354-1356.

51. Krishnan S, Mathuru AS, Kibat C, et al. The right dorsal habenula limits attraction to an odor in zebrafish. Curr Biol. 2014; 24(11): 1167-1175.

52. Facchin L, Duboue ER, Halpern ME. Disruption of Epithalamic LeftRight Asymmetry Increases Anxiety in Zebrafish. J Neurosci. 2015; 35(48): 15847-15859.

53. Lagadec R, Laguerre L, Menuet A, et al. The ancestral role of nodal signalling in breaking $\mathrm{L} / \mathrm{R}$ symmetry in the vertebrate forebrain. Nat Commun. 2015; 6: 6686 .

54. Signore IA, Palma K, Concha ML. Nodal signalling and asymmetry of the nervous system. Philos Trans R Soc Lond B Biol Sci. 2016; doi: $10.1098 /$ rstb.2015.0401.

55. Concha ML, Burdine RD, Russell C, et al. A nodal signaling pathway regulates the laterality of neuroanatomical asymmetries in the zebrafish forebrain. Neuron. 2000; 28(2): 399-409.

56. Roussigne M, Bianco IH, Wilson SW, et al. Nodal signalling imposes left-right asymmetry upon neurogenesis in the habenular nuclei. Development. 2009;136(9):1549-1557.

57. Grande C, Martin-Duran JM, Kenny NJ, et al. Evolution divergence and loss of the Nodal signalling pathway new data and a synthesis across the Bilateria. Int J Dev Biol. 2014; 58(6-8): 521-532.

58. Shiratori $\mathrm{H}$, Hamada $\mathrm{H}$. TGFbeta signaling in establishing left-right asymmetry. Semin Cell Dev Biol. 2014; 32: 80-84.

59. Gamse JT, Thisse C, Thisse B, et al. The parapineal mediates left- 
right asymmetry in the zebrafish diencephalon. Development 2003;130(6):1059-1068.

60. Concha ML, Signore IA, Colombo A. Mechanisms of directional asymmetry in the zebrafish epithalamus. Semin Cell Dev Biol. 2009; 20(4): 498-509.

61. Aizawa H, Goto M, Sato T, et al. Temporally regulated asymmetric neurogenesis causes left-right difference in the zebrafish habenular structures. Dev Cell. 2007;12(1): 87-98.
62. Husken U, Stickney HL, Gestri G, et al. Tcf7l2 is required for left-right asymmetric differentiation of habenular neurons. Curr Biol. 2014; 24(19): 2217-2227.

63. Signore IA, Concha ML. Heterochrony and Morphological Variation of Epithalamic Asymmetry. The Journal of Experimental Zoology Part B. 2016;doi: 10.1002/jez.b.22698.

64. Rogers LJ, Vallortigara G, Andrew RJ. Divided Brains. Cambridge: Cambridge University Press; 2013. 